\title{
NEW DEVELOPMENTS IN CATIONIC AND ANIONIC ALDEHYDE POLYMERIZATION
}

\author{
P. Kubisa, I. Negulescu, K. Hatada, D. Lipp, J. Stark, B. Yamada and O. Vogl \\ Polymer Science and Engineering, University of Massachusetts, Amherst, MA 01002, USA
}

\begin{abstract}
Aldehydes polymerize readily with cationic and anionic initiators to polyacetals. Higher aliphatic aldehydes are conveniently polymerized with anionic initiators at low temperatures, frequently to isotactic polyaldehydes. The range of known polyaldehydes has recently been extended to poly ( $n$-dodecaldehyde). Polymers with side chains longer than $\mathrm{C}_{3}$ possess the characteristics of the crystallization of the aliphatic side chain separate and in addition to the crystallization of the main chain.

An NMR investigation of the rate of polymerization and copolymerization of chloral showed that the polymerization becomes diffusion controlled above approximately $40 \%$ conversion. At that conversion small molecules are entrapped in the polymer matrix and individual parts of these molecules have different mobility as may be detected by NMR.

Bromal has recently been polymerized at low temperatures where its crystallization was avoided by judicious selection of solvent and temperature of polymerization. The tribromomethyl group of bromal is the largest side group of any monomer which has ever been polymerized. Dichlorobromoacetaldehyde and chlorodibromoacetaldehyde were synthesized and polymerized to what are completely insoluble and apparently isotactic polymers. Fluorodichloroacetaldehyde and difluorochloracetaldehyde were also synthesized and polymerized. Fluorodichloroacetaldehyde gives only insoluble and apparently isotactic polymers. Difluorochloroacetaldehyde gave soluble amorphous polymers in addition to crystalline polymers.

Future trends in aldehyde and carbonyl polymerization are discussed and attempts have been made to identify unsolved problems in this area. The successful preparation of tetrachloroethylene oxide, in good yield, has been disclosed together with the failure of all attempts to homopolymerize this compound or copolymerize it with potential comonomers.
\end{abstract}

\section{PRESENT STATE OF ALDEHYDE POLYMERIZATION}

Aldehydes are polymerized with ionic initiators to polyacetals $^{1-3}$ but with a much lower $\Delta \mathrm{H}_{\text {polym. }}$ and consequently also a lower ceiling temperature than when $\alpha$-olefins of comparable structure are polymerized to polyolefins. In ethylene $\left(\mathrm{CH}_{2} \mathrm{CH}_{2}\right)$ which is compared with $\left(\mathrm{CH}_{2} \mathrm{O}\right)$ formaldehyde, the carbonyl oxygen is formally replaced by a terminal $\mathrm{CH}_{2}$ group. Consequently, propylene $\left(\mathrm{CH}_{3} \mathrm{CHCH}_{2}\right)$ should also be compared with acetaldehyde $\left(\mathrm{CH}_{3} \mathrm{CHO}\right)$.

By far the most important polyaldehyde is polyoxymethylene (polyformaldehyde). It is now commercially produced by anionic polymerization of purified formaldehyde. $^{4,5}$ The initiation of this polymerization is often carried out by an acylate and the propagation of this polymerization is via the growing alkoxide anion. Since the acylate is not a very efficient initiator for formaldehyde polymerization, much of the polymerization is dominated by chain transfer to chain transfer agents present in the monomer, for example, water and alcohols.

$$
\begin{aligned}
& \mathrm{R}^{\ominus}+\mathrm{CH}_{2} \mathrm{O} \longrightarrow \mathrm{R}-\mathrm{CH}_{2}-\mathrm{O}^{\ominus} \\
& \mathrm{R}-\mathrm{CH}_{2}-\mathrm{O}^{\ominus}+n-\mathrm{CH}_{2} \mathrm{O} \longrightarrow \mathrm{R}-\left(\mathrm{CH}_{2}-\mathrm{O}\right)_{n}-\mathrm{CH}_{2}-\mathrm{O}^{\ominus}
\end{aligned}
$$

Cationic polymerization of formaldehyde may also be carried out but polymers of relatively high molecular weight (polyoxymethylene) can only be prepared with cationic initiators which have very stable anions because of the ease of chain termination by recombination with anion fragments. Cationic initiation, propagation and termination of formaldehyde polymerization can be described in a very similar manner to anionic polymerization. ${ }^{6}$

$$
\begin{aligned}
& \mathrm{R}^{\oplus}+\mathrm{O}=\mathrm{CH}_{2} \longrightarrow \mathrm{R}-\mathrm{O}^{\oplus}=\mathrm{CH}_{2} \\
& \mathrm{R}-\mathrm{O}^{\oplus}=\mathrm{CH}_{2}+n-\mathrm{OCH}_{2} \longrightarrow \mathrm{R}+\mathrm{O}-\mathrm{CH}_{2}+\mathrm{O}^{\oplus}=\mathrm{CH}_{2}
\end{aligned}
$$

Anionic copolymerizations of formaldehyde could be very conveniently carried out with monomers which could undergo anionic homopolymerization such as isocyanates and ketenes, while cationic copolymerization of formaldehyde has been reported with compounds which normally undergo cationic homopolymerization such as vinyl ethers and cyclic ethers.

The discussion of the polymerization of substituted aldehydes is most conveniently divided into the polymerization of higher aliphatic aldehydes, including the cyclopolymerization of aliphatic aldehydes, and the polymerization of haloaldehydes. Higher aliphatic aldehydes may be polymerized with anionic and cationic initiators but require strong nucleophiles such as alkoxides while relatively weak electrophiles are effective. ${ }^{7}$ For the initiation of the polymerization of haloaldehydes, weak nucleophiles may be, but strong electrophiles must be used, to be effective. It should also be pointed out that in essentially all these polymerizations, the polymer precipitates during the polymerization as a crystalline polymer which adds an additional driving force for the polymerization.

The most extensively studied aldehyde other than formaldehyde is acetaldehyde. It was an important 
discovery that amorphous polyacetaldehyde ${ }^{8}$ must be made at low temperature and provided a sound experimental basis for the principal of ceiling temperature. Subsequent work showed that the low temperature polymerization of acetaldehyde gave amorphous, atactic or crystalline isotactic forms of polyacetaldehyde with classical cationic initiators, Lewis acids, such as $\mathrm{BF}_{3}$, or anionic initiators, such as alkali metal alkoxides respectively. It was found at the same time that aluminum alkyls, or aluminum alkyls modified by reaction with alcohols, amines and other organic compounds, could give highly effective initiators for acetaldehyde polymerization and produced initiators which were capable of polymerizing acetaldehyde to highly isotactic polymers. ${ }^{9}$ Modified aluminum alkyls and zinc alkyls are not only good initiators for the polymerization of acetaldehyde and higher aliphatic aldehydes but are very effective for the polymerization of ethylene oxide, propylene oxide and other cyclic ethers, thio ethers, etc. lactones and lactams. ${ }^{2}$

Aliphatic aldehyde polymers became increasingly regular as the length of the side group increased from acetaldehyde to $n$-butyraldehyde. The phenomenon of a dual melting point was noticed starting with the crystalline $n$-valeraldehyde polymer, but is particularly pronounced in the case of $\operatorname{poly}\left(n\right.$-heptaldehyde). ${ }^{10}$

A further development in aldehyde polymerization was the systematic study of the polymerization of aliphatic aldehydes with polar side groups which is still proceeding. ${ }^{11}$ These monomers were predominantly addition products of acrolein, for example, the nitriles, ethers, thio ethers and esters of beta substituted propionaldehydes.

Dialdehydes with the proper spacing of 2-3 carbon atoms between the aldehyde groups could be cyclopolymerized, as, for example, in the case of succinaldehydes, glutaraldehydes, but also in the case of unsaturated and aromatic dialdehydes, for example, malealdehyde and phthalaldehyde. This ability to polymerize aromatic and unsaturated dialdehydes is in contrast to the fact that, aromatic monoaldehydes, such as benzaldehydes, where the aldehyde group is directly attached to the aromatic ring, cannot be polymerized.

Polymers of haloaldehydes have also been studied in the past, particularly the polymerization of chloral. A significantly new development of recent years is the discovery of the cryotachensic polymerization of chloral which allows the preparation of films and sheets, the fabrication of the infusible and insoluble chloral polymer, by monomer casting. . $^{12,13}$

Most haloaldehyde polymers are polymers of substituted acetaldehydes which are halogenated to various extents because compounds with fully halogenated longer aliphatic chains cannot be prepared with the exception of perfluoro carbonyl compounds. Examples of perfluoroaldehydes are known and their polymerization has been described. Fluoral has been polymerized to crystalline, presumably isotactic, polyfluoral and amorphous, presumably atactic, fluorocarbon soluble polyfluoral. The kinetics and the thermodynamic behavior of fluoral polymerization have also been studied. ${ }^{14,15}$

At the present time aldehyde polymerization is studied in the following areas: (a) the polymerization and copolymerization of aldehydes with normal aliphatic side chains, (b) the preparation of aliphatic aldehyde polymers with various functional groups, (c) more extended studies of chloral polymerization and (d) the preparation and polymerization of perhalogenated acetaldehydes including acetaldehydes with $\mathrm{F}, \mathrm{Cl}$ and $\mathrm{Br}$ substitution.

\section{POLYMERS OF HIGHER ALIPHATIC ALDEHYDES}

\section{A. Structural characteristics of polymers of high aliphatic} aldehydes

We had earlier recognized that crystalline polymers of $n$-valeraldehyde, $n$-heptaldehyde and $n$-octaldehyde showed the behavior of a "double melting point". 16,17 It appeared reasonable to suggest that the higher melting point (near $150^{\circ} \mathrm{C}$ ) was associated with the melting of the polyoxymethylene backbone chain (equilibrium melting point of polyoxymethylene is $178^{\circ} \mathrm{C}$ ), and the lower melting point was associated with the melting of a separately crystallized phase of the waxy aliphatic side chains.

We have now investigated more thoroughly the preparation and the melting behavior of the $C_{5}$ to $C_{8}$ aldehyde polymers, with particular emphasis on the behavior of crystallization and melting of the aliphatic side chain. Crystalline poly ( $n$-butyraldehyde) shows only one melting point near $245^{\circ} \mathrm{C}$. Poly $(n$-valeraldehyde) did not show distinctly the behavior of side chain crystallization. Much of our work was done with $\operatorname{poly}(n-$ heptaldehyde) because this polymer shows this behavior most clearly (Fig. 1).

$n$-Heptaldehyde was polymerized in methylcyclohex-

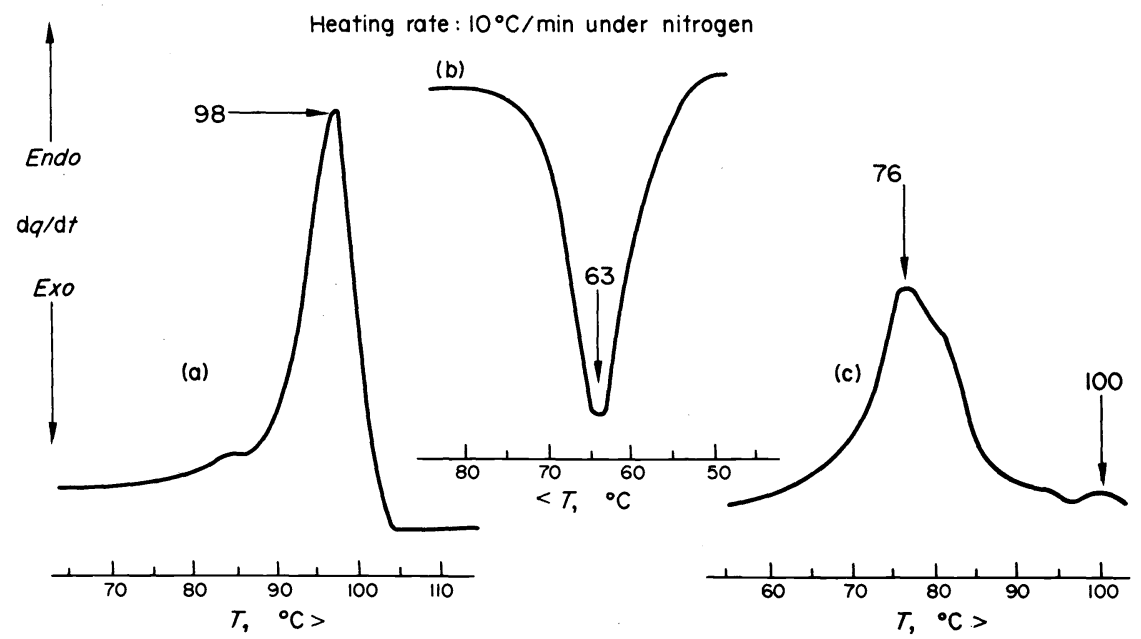

Fig. 1. Poly( $n$-heptaldehyde): Side chain crystallization, DSC studies. 
ane at $-78^{\circ}$ with lithium tertiary butoxide as the initiator. The polymer was acetate capped with a mixture of acetic anhydride/pyridine and was of moderate molecular weight $(\sim 10,000)$ as judged by osmometric determination. $\operatorname{Poly}(n$-heptaldehyde) (PHA) was highly crystalline and according to $300 \mathrm{MHz}$ PMR spectroscopy, it was isotactic.

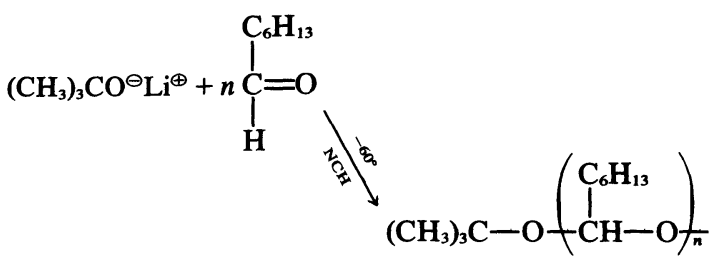

Transition regions which could be attributed to side chain crystallization were in the range of $75-105^{\circ}$. At least two transitions can be identified, one at $77-79^{\circ} \mathrm{C}$ and $103-105^{\circ} \mathrm{C}$ with another probable one at $86-87^{\circ} \mathrm{C}$. Figure 1 shows this region in PHA "as obtained" and after recrystallization. It can be seen that the low temperature
$C_{7}$ (n-octaldehyde) have not yet been successfully polymerized. The failure for their polymerization could be traced to the relatively higher crystallization rate of the aldehyde as compared with its rate of polymerization. Several reports from the literature indicate that aliphatic aldehydes with longer aliphatic side chains show peculiar melting point behavior. It is apparent that association of the aldehyde molecules occurs in solution and perhaps even a liquid crystal type solution is formed under certain conditions. This might be the reason why crystallization rather than polymerization of these aldehydes occurs even in the presence of the initiator. Where polymerization temperature and temperature of crystallization are similar, very accurate temperature conditions must be maintained in order to accomplish polymerization rather than the crystallization of the aliphatic aldehyde.

When optimum conditions were chosen with lithium tertiary butoxide as the initiator in methylcyclohexane, $n$-dodecylaldehyde, polymerized to crystalline poly $(n$ dodecylaldehyde) in reasonable yields. The polymer could be endcapped and showed the following analysis. Elemental analysis: Calcd. for $\left(\mathrm{C}_{12} \mathrm{H}_{24} \mathrm{O}\right), \mathrm{C}, 78.28$; $\mathrm{H}$, 12.91. Found: C, 78.76; H, 12.80 .

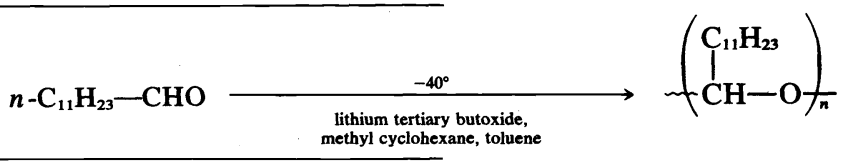

transition at $76^{\circ} \mathrm{C}$ increased remarkably at the expense of the $103^{\circ} \mathrm{C}$ transition after slow crystallization.

Additional information was obtained by studying the transition region by extrusion just above the "melting" of the side chain crystallinity, and after annealing, and comparing these results with measurement of the original polymers (Table 1).

The crystallinity depended very much on the history of the polymer. Mechanical treatment effected the backbone orientation but also the crystallization of the side group. Extruded poly( $n$-heptaldehyde)(PHA-E) showed a much lower value of side chain crystallinity as compared with non-extruded material. The crystallinity and the apparent size of crystallites are related to the temperature of the DSC transitions and their contribution to the total heat of fusion, $\Delta \mathrm{H}_{\mathrm{f}}$.

\section{B. Poly(n-dodecylaldehyde)}

Higher aliphatic aldehydes with side chains longer than
Poly( $n$-dodecylaldehyde) was allowed to react with an aqueous/alcoholic solution of 2,4-dinitrophenyl hydrazine in the presence of sulfuric acid and gave a high yield of the 2,4-dinitrophenylhydrazone of $n$-dodecylaldehyde with a melting point of $104-107^{\circ} \mathrm{C}\left(\mathrm{Lit} .=106^{\circ} \mathrm{C}\right) .^{18}$

Poly $(n$-dodecylaldehyde) was soluble in chloroform and the i.r. spectrum could be measured in chloroform solution as well as for the neat compound. The polymer was cast onto a sodium chloride plate from $\mathrm{CDCl}_{3}$ solution and the solvent evaporated. It can be seen from this spectrum (Fig. 2) that the carbonyl band of the initial monomer, $n$-dodecanal, is not present and only a small amount of carbonyl absorption attributable to the acetate end group is noticeable. In addition, the carbon hydrogen bands between 3000 and $2800 \mathrm{~cm}^{-1}$ are strong but the $\mathrm{C}-\mathrm{O}-\mathrm{C}$ absorptions in the $1200-1050 \mathrm{~cm}^{-1}$ are relatively weak as one would expect from the structure of this poly( $n$-dodecylaldehyde).

The transitions of this polymer were measured in a DSC-1B Perkin-Elmer instrument at a heating rate of

Table 1 . Side-chain crystallinity of oriented and unoriented poly( $n$-heptaldehyde $) \dagger$

\begin{tabular}{|c|c|c|c|c|c|c|}
\hline \multirow{4}{*}{$\begin{array}{l}\text { Polymer } \\
\text { PHA } \\
\text { PHA-EA } \\
\text { PHA-EA }\end{array}$} & \multirow{2}{*}{$\begin{array}{l}\begin{array}{l}\text { Thermal } \\
\text { history }\end{array} \\
\text { Unoriented }\end{array}$} & \multicolumn{2}{|c|}{$\begin{array}{c}\text { Temperature } \\
\text { of DSC peaks } \\
\left({ }^{\circ} \mathrm{C}\right)\end{array}$} & \multirow{2}{*}{$\begin{array}{c}\begin{array}{c}\Delta \mathbf{H}_{\mathrm{f}} \\
(\mathrm{cal} / \mathrm{g})\end{array} \\
8.4\end{array}$} & \multirow{2}{*}{$\begin{array}{c}\begin{array}{c}\text { Side chain } \\
\text { crystallinity } \\
(\%)\end{array} \\
21\end{array}$} & \multirow{2}{*}{$\begin{array}{l}\text { Contribution of } \\
\text { the last DSC peak } \\
\text { for } \Delta \mathrm{H}_{\mathrm{f}}(\%)\end{array}$} \\
\hline & & 86 & 99 & & & \\
\hline & Extruded & 77 & 104 & 3.3 & 8 & 16 \\
\hline & $\begin{array}{l}\text { Annealed at } \\
93^{\circ}, 4 \mathrm{hr}\end{array}$ & 75 & 103 & 6.3 & 16 & 20 \\
\hline PHA-EA & $\begin{array}{l}\text { Annealed at } \\
93^{\circ}, 10 \mathrm{hr}\end{array}$ & 76 & 104 & 7.3 & 18 & 33 \\
\hline PHA & Reference & 87 & 101 & 7.3 & 18 & 61 \\
\hline PHA-A & $\begin{array}{l}\text { Annealed at } \\
90^{\circ}, 2 \mathrm{hr}\end{array}$ & 78 & 103 & 7.1 & 18 & 44 \\
\hline PHA-A & $\begin{array}{l}\text { Annealed at } \\
90^{\circ}, 10 \mathrm{hr}\end{array}$ & 79 & 105 & 7.4 & 19 & 59 \\
\hline
\end{tabular}

$\dagger$ Poly $\left(n\right.$-heptaldehyde),(PHA) was oriented by extrusion in INSTRON,PHA-E, at $100^{\circ} \mathrm{C}$ and $5 \mathrm{~cm} / \mathrm{min}$, through a capillary of $\mathrm{d}=0.5 \mathrm{~mm}$ and $1=25.4 \mathrm{~mm}$. 


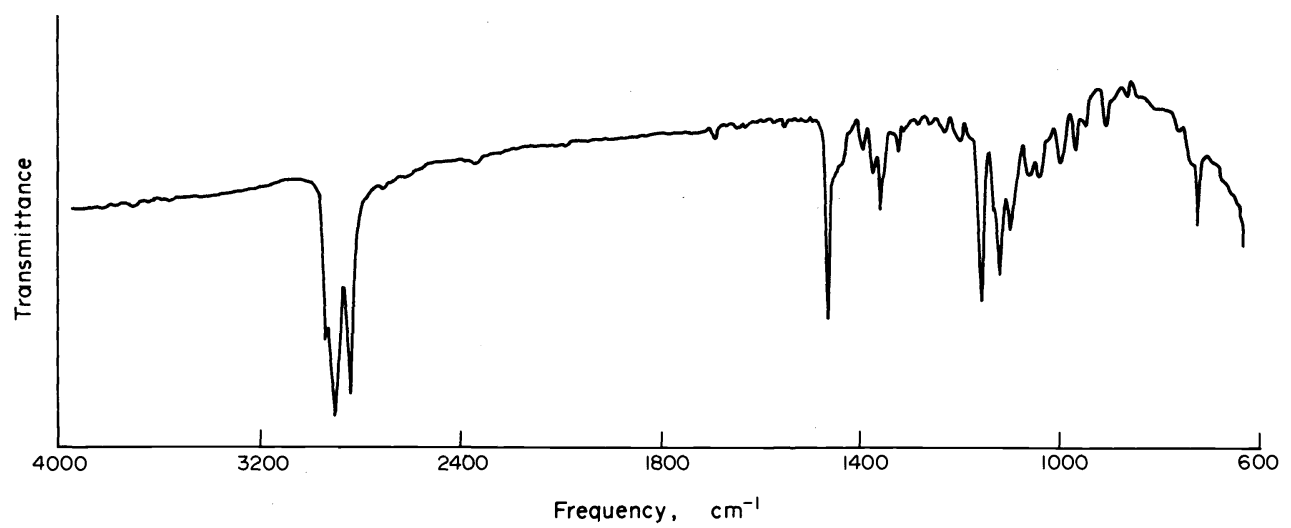

Fig. 2. Poly( $n$-dodecylaldehyde): I.R. spectrum, neat on $\mathrm{NaCl}$.

$20^{\circ} \mathrm{C} /$ min under nitrogen (Fig. 3). In the first scan two transition peaks in the region of $50-70^{\circ} \mathrm{C}$ were observed. The first peak had a maximum at $59^{\circ} \mathrm{C}$ and the second peak at which was much more prominent $69^{\circ} \mathrm{C}$. After slowly cooling below the transition region and rescanning, only one peak was observed at $60^{\circ} \mathrm{C}$. This is apparently the first peak which was observed in our first scanning. Although these results are of a preliminary nature, it shows clearly that the transition behavior of the side chain crystallization is qualitatively similar to that of the behavior of poly $(n$-heptaldehyde) where the transition region consists of at least three responses. Peak heights and transition temperatures depend on the thermal history of the poly $(\boldsymbol{n}$-dodecylaldehyde) sample.

The thermal degradation of poly $(n$-dodecylaldehyde) was also measured using a TGS-1 Perkin-Elmer thermal balance; the maximum degradation temperature of this sample was $274^{\circ} \mathrm{C}$ at a heating rate of $20^{\circ} \mathrm{C} / \mathrm{min}$.

\section{HALOALDEHYDE POLYMERS}

\section{A. Polychloral}

Chloral can be polymerized most effectively and rapidly with anionic initiators. ${ }^{12}$ When the polymerization was
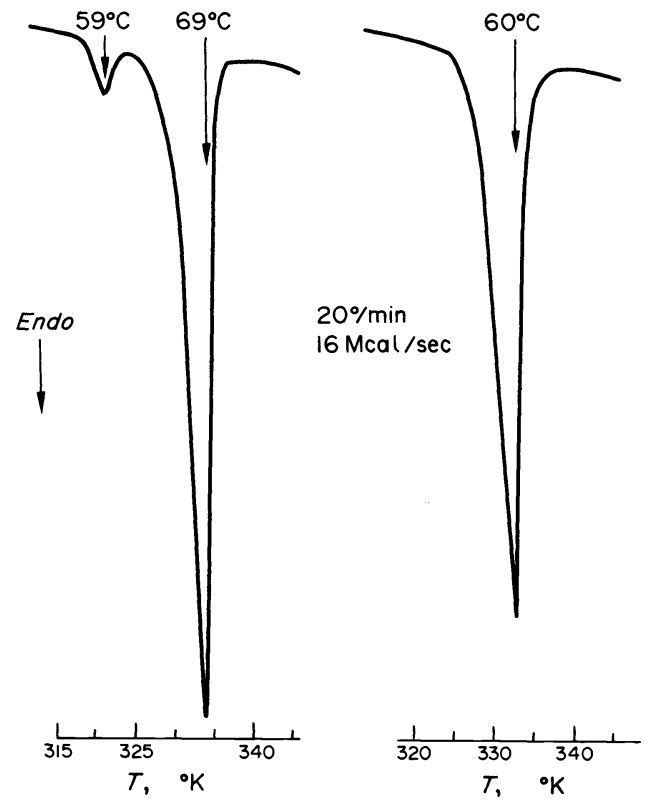

Fig. 3. Poly(n-dodecylaldehyde): Side chain crystallization DSC studies. carried out under cryotachensic conditions, by initiation of bulk monomer above the threshhold temperature of polymerization, uniform pieces of polychloral are obtained in about $85 \%$ yield. The yield limitation is apparently not caused by the attainment of equilibrium between polymer and monomer but rather because the monomer is unable to diffuse to the reaction site. It has been found that polymerization of chloral in the presence of about $20 \%$ of an inert solvent, such as hexane, increased the mobility of the monomer and yields of more than $96 \%$ of polychloral have been obtained.

Rate of polymerization. We carried out rather extensive studies of the progress of the homopolymerization of chloral and its copolymerization with phenyl isocyanate by PMR spectroscopy in which the disappearance of chloral in the case of homopolymerization and the disappearance of chloral and comonomer in the case of copolymerization was determined. The rate of polymerization depends primarily on the type and amount of initiator used and is faster with increasing amounts of initiator. Since polychloral precipitated as an apparently homogeneous gel, as polymerization proceeded, the rate of polymerization decreased as the conversion increased.

The effectiveness of anionic initiators falls primarily in two main categories, those that react immediately and to a high degree with one mole of chloral to form the initiating alkoxide,

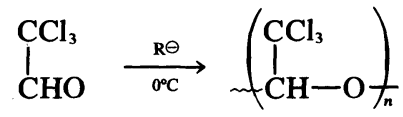

and those whose initiation equilibrium does not favor the addition of the nucleophile to the electrophilic carbonyl carbon atom of chloral.

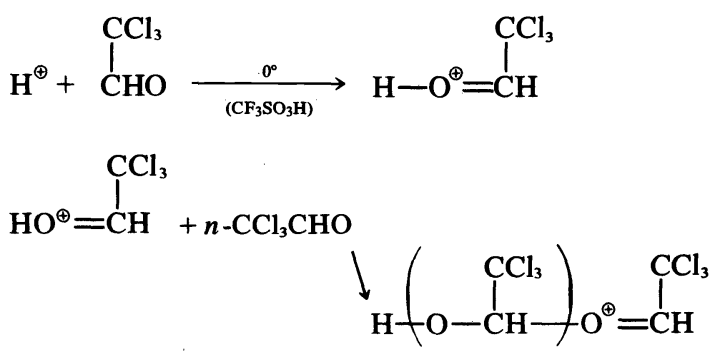

Lithium tertiary butoxide reacts essentially quantitatively with chloral to form an alkoxide which can be 
readily detected by proton resonance of the acetalic hydrogen at approximately $5 \mathrm{ppm}$. The other group of initiators which includes tertiary aromatic amines showed no significant reaction with one mole of chloral and no proton resonance was found at $5 \mathrm{ppm}$.

Triphenylphosphine was the most extensively studied initiator in our rate study of chloral polymerization. Triphenylphosphine reacted almost instantaneously with one mole of chloral to form triphenyl dichlorovinyloxy phosphonium chloride. As a consequence, the actual initiator for chloral polymerization is the chloride ion. It can be seen from Fig. 4 that the polymerization of chloral with triphenylphosphine goes to completion in 5-15 min depending on the initiator concentration. At $0.1 \mathrm{~mol} \%$ initiator concentration the polymerization is slow and proceeds at an initial rate of about $14 \% / \mathrm{min}$ while at $0.3 \mathrm{~mol} \%$ the initial rate is about $33 \% / \mathrm{min}$.

With lithium tertiary butoxide as the initiator, the polymerization is much faster and even at $0.1 \mathrm{~mol} \%$ initiator concentration the polymerization goes to completion as fast as the PMR measurements can be made.

Other compounds which polymerize chloral belong to the group of slower initiators. As compared with triphenylphosphine, pyridine is a slightly faster initiator and tetrabutyl ammonium chloride is somewhat slower but $\gamma$-collidine is even slower than tributylammonium chloride for the polymerization of chloral using the same amount of initiator.

Interestingly enough, the rate of chloral polymerization is practically unaffected by the kind of diluent used for the polymerization. Aromatic and aliphatic hydrocarbons in up to $10-15 \%$ of diluent show similar rates. This is not really surprising because chloral monomer is much more polar than the inert solvents and is probably strongly associated and solvates the growing polymer. The relative rate of disappearance of chloral and comonomer during the copolymerization of chloral and isocyanates depends greatly on the type of isocyanates used for the copolymerization. Aromatic isocyanates are more readily incorporated into the copolymer as seen in Fig. 5.

Mobility of small molecules in polychloral matrix. In the course of our rate study of chloral polymerization by PMR spectroscopy it was found that broadening of

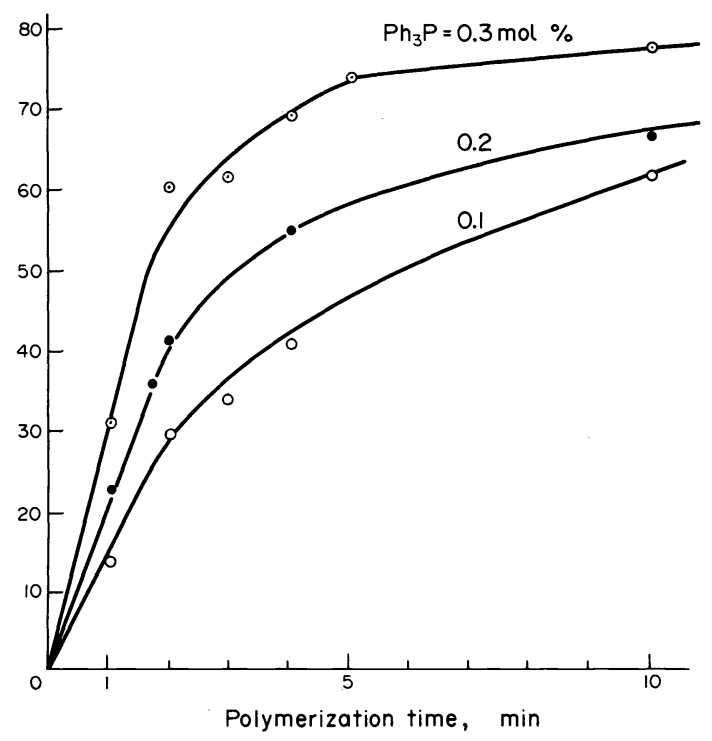

Fig. 4. Chloral polymerization: Rate study by NMR.

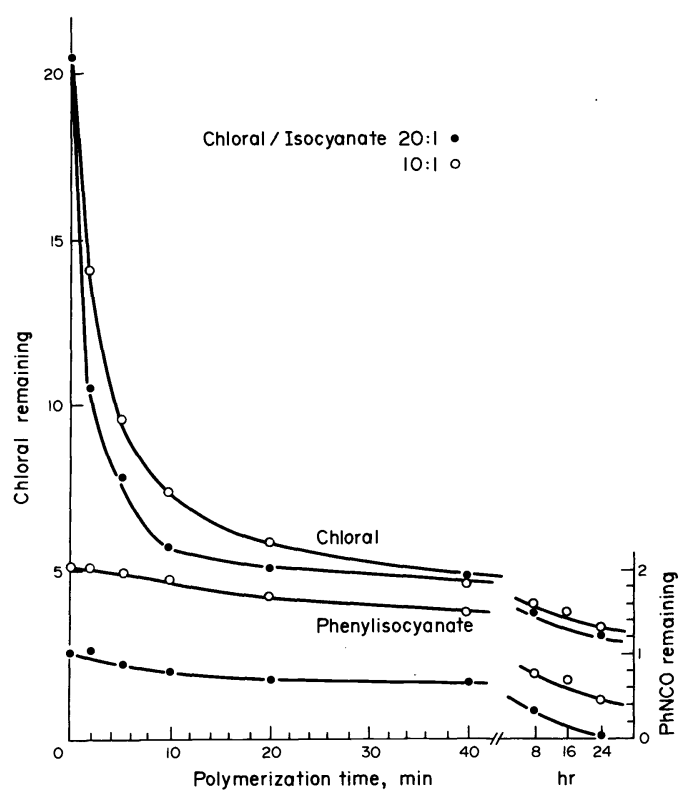

Fig. 5. Polymerization of chloral with phenylisocyanate: Rate study by NMR.

the line width of the PMR signals of chloral as well as that of various solvents was observed as the conversion of chloral to polychloral increased. ${ }^{19}$

The line width of the solvents increased depending on the type of groups attached to the proton which is responsible for the line broadening. The chloral signal showed only a slight increase in the signal broadening during polymerization even at high conversion. In the case of toluene, the line broadening was very substantial especially in the aromatic proton region as compared with the signal broadening of the protons of aliphatic substituents. The line broadening is believed to be a spin-spin relaxation process in our system arising from time correlation functions of magnetic dipole interactions among all hydrogen atoms. Since the signals were close to Laurentzian, the spin-spin relaxation time, $T_{2}$, could be obtained from the spectra line width. However, various complications, particularly the inhomogeneity of the magnetic field and possible inhomogeneity of the polychloral gel, did not allow us to make accurate estimates of $T_{2}$. As a consequence, the line width $\left(\Delta \mathrm{H} / \mathrm{H}_{0}\right)$ was used as the measure of the mobility of the small molecules in the polychloral matrix. When the ratio between the line width of chloral and also of other small molecules used as solvents, is plotted against the conversion of chloral to polychloral, curves were obtained which showed that aromatic groups are more readily broadened than some aliphatic groups especially if they are situated at the end of the molecule as is the case of toluene (Fig. 6).

It is believed that both chloral and the solvents are entrapped in the polychloral matrix. However, aromatic hydrocarbons are apparently more strongly entrapped than chloral. This behavior seems to indicate that there is a different degree of interaction between the trichloromethyl groups of the polychloral which are protruding from the polymer backbone chain and individual parts of small molecules, and cause an interaction of various degrees with small molecules. With aromatic compounds, there is a possible charge transfer type interaction between the aromatic ring and the trichloromethyl group which 


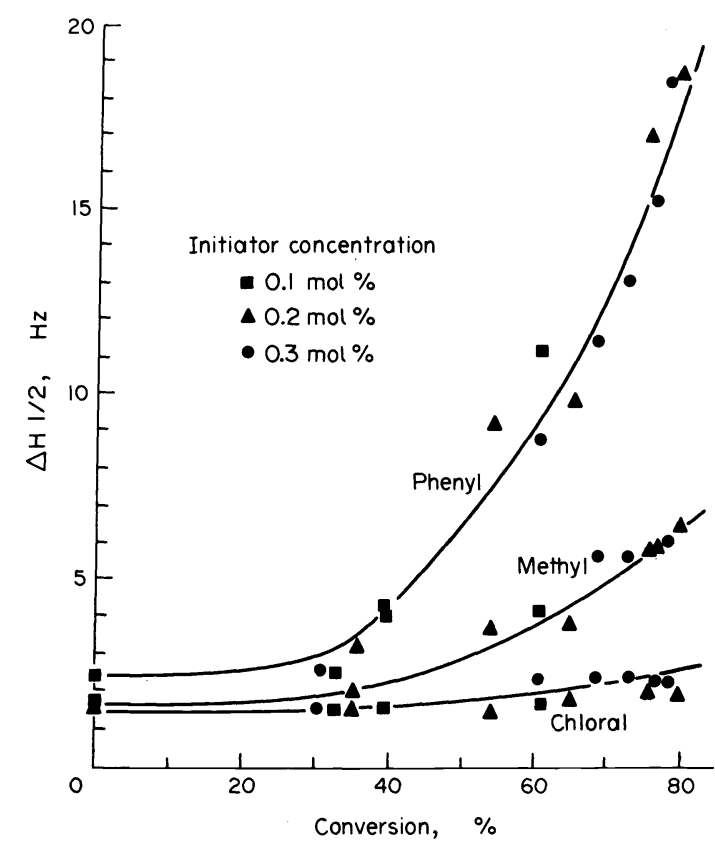

Fig. 6. Polychloral: PMR line broadening of toluene and chloral signals.

prevents full mobility of the aromatic ring at high conversions; the-CHO group of chloral is essentially free.

Stabilization of chloral homopolymer by chain transfer/termination reactions. Chloral homopolymer prepared by anionic polymerization is relatively unstable. It cannot be acetylated with acetic anhydride under the normal conditions apparently because of the inability of the acetylating reagents, acetic anhydride, to penetrate to the reaction site..$^{20}$

The end groups of chloral homopolymers have still not been identified because they have no characteristic absorption in the infrared spectrum. Since polychloral is insoluble and infusible, only a limited amount of characterization, most of which relies on some solution techniques, is possible. The determination of the thermal degradation spectrum is one of the major means of characterizing chloral polymers and distinguishing them from one another. Polychloral samples prepared with different initiators under different reaction conditions give distinct and characteristic spectra of polymer stability. In general, polychloral samples prepared by anionic polymerization, are less stable than those prepared by cationic polymerization. From comparative studies of anionic and cationic polymers and polymers which have been stabilized in various ways (for example, by treatment with $\mathrm{PCl}_{5}$ ). The chemical nature of the stabilization is not always known and a characterization of individual polymer samples can be made by the measurement of its thermal degradation spectrum (Fig. 7).

It was believed possible to improve the stability of polychloral samples and possibly prepare polychloral of ultimate thermal stability by carrying out the polymerization in the presence of acetylating agents. We have indeed found that anionic chloral polymerization can be carried out in the presence of acetyl chloride or benzoyl chloride if the polymerization is initiated by triphenylphosphine. Up to $2 \mathrm{~mol} \%$ of acetyl chloride could be added to the polymerization mixture without interferring with the polymerization and the conversion of monomer to polymer is similar with or without acetylating agents Lithium tertiary butoxide could not be used as the initiator because it reacted immediately with the acetylating agent which prevented the polymerization.

During this polymerization there is an apparent competition between the addition of chloral and acetyl chloride to the growing polymeric alkoxide.

However, very little acetyl chloride reacted during the polymerization and only after the completion of the polymerization, in a much slower reaction, acetylation of the completed polymer was observed. The progress of the acetylation can be readily followed by the determination of the i.r. spectrum of the final polymer

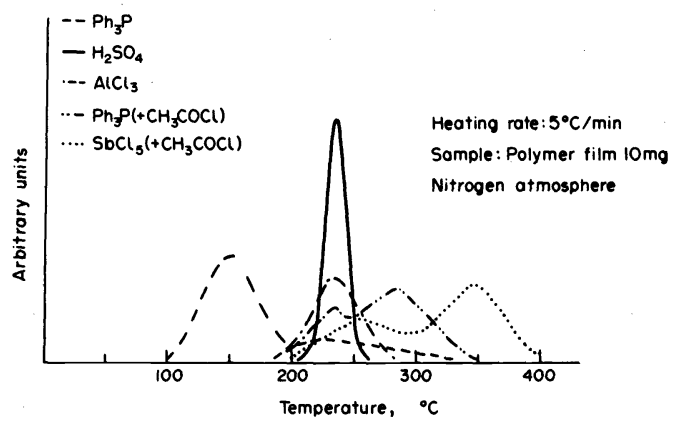

Fig. 7. Polychloral: Thermal stability (DTG) of various samples.<smiles>CC(=O)OC(P)C(F)(Cl)Cl</smiles> 
(Fig. 8) and further confirmed by a change in the thermal degradation spectrum, the appearance and increase of a peak of the DTG curve at higher temperatures, indicating the production of a more stable polychloral fraction (Fig. 9).

This result seems to indicate that chloral polymerization initiated by triphenylphosphine (triphenyl divinyloxy phosphonium chloride) in the presence of limited amounts of acylating agents proceeds first in a fast reaction to a polychloral with alkoxide end groups that become occluded in the later part of the reaction. This has two consequences: (1) The monomer cannot penetrate to the reactive site which leads to a cessation of the polymerization at $85 \%$ conversion. (2) When acylation is attempted in a second reaction, the reagent apparently cannot diffuse to the reaction site and the acylation of the alkoxide does not succeed. On heating this unstable polymer, it will, however, degrade rather than being acetylated. If the acylating agent is, however, part of the polymerization mixture, it remains in close vicinity to the occluded but still reactive alkoxide and can then undergo the desirable acylation reaction (Fig. 10). The results of our investigation showed that one-half of the reactive sites could be acylated under our present reaction conditions. Additional work, perhaps the use of an acylating or an alkylating group as part of the counter cation, would be a more efficient way to carry out this reaction.

\section{B. Cationic polymerization}

Early work on the polymerization of chloral has shown that aluminum chloride and sulfuric acid can act as initiators. We carried out additional work in attempts to determine the scope of the cationic polymerization of chloral. Many commonly used Lewis acids, cationic salts and other cationic initiators

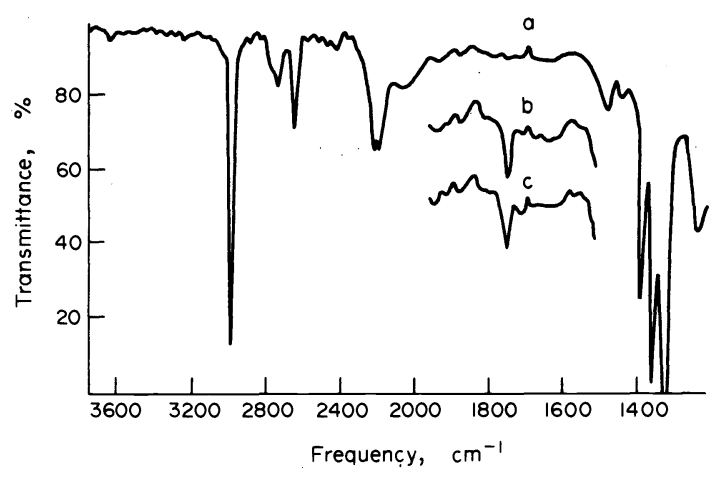

Fig. 8. Polychloral: End group identification by i.r.

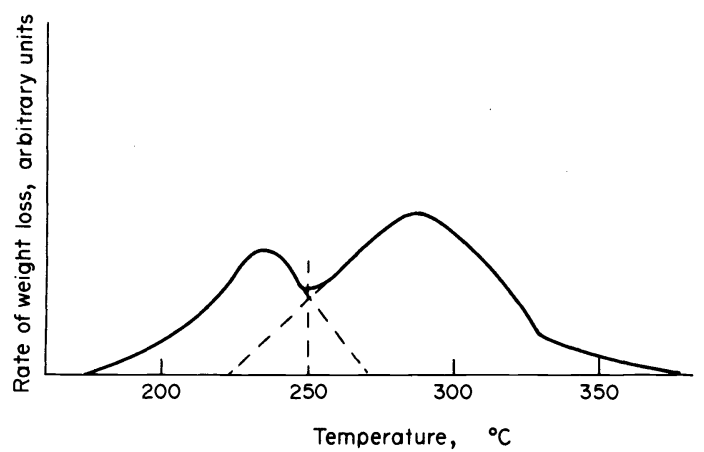

Fig. 9. Polychloral: DTG curve of polymer in the presence of acylating agent.

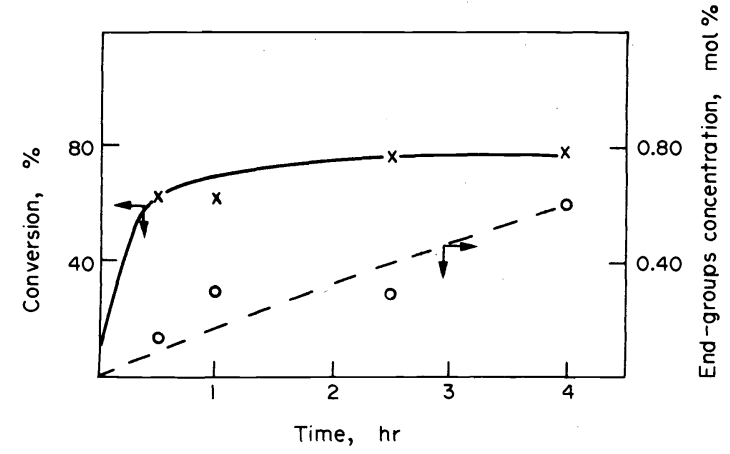

Fig. 10. Polychloral: Progress of acylation.

have, however, proved to be ineffective. We have confirmed that aluminum chloride is indeed an adequate initiator for chloral polymerization but an equally good initiator was trifluoromethyl sulfonic acid. ${ }^{21}$ In both cases, the polymerization did not proceed in the high rate which was established for the case of anionic polymerization and the morphology of the polymer observed was different from the gel-like character of anionic polymers.

$\mathrm{SbCl}_{5}$ or $\mathrm{SbCl}_{5}$ with $5 \mathrm{~mol} \%$ of acetyl chloride also gave polychloral in modest yields after 3 days of reaction time. This latter combination and the establishment of trifluoromethyl sulfonic acid as a good initiator established firmly the cationic polymerization of chloral. Other initiators such as ferric chloride and titanium tetrachloride gave only a few per cent yield of polymer after several days of reaction while most other Lewis acids were ineffective.

\section{Other perhaloaldehyde polymers}

Chloral and fluoral polymers are the only well investigated perhaloaldehyde polymers. Fluoral may be prepared as a crystalline and presumably isotactic polymer or as an amorphous and soluble, presumably atactic polymer. Polychloral has only been prepared in one form, the crystalline insoluble, isotactic polymer. The only other perhaloacetaldehyde polymer which was described briefly in a patent was an insoluble polymer from difluorochloroacetaldehyde.

We have undertaken a more thorough study of the preparation of the unknown perhaloacetaldehydes with more than one halogen substituted in the perhalomethyl methyl group in an attempt to study their polymerization or at least to determine their polymerizability. When polymerization was established, it was found desirable to determine whether the polymer could be made in stereoregular and/or in atactic form, in order to find out if size and shape of the side chain had any influence upon the stereoregulation of polymer formation.

Unlike olefins and vinyl ethers which require transition metal initiators whose metal atom must have the proper ligand structure and stability of the coordination complex in order to produce stereoregular polymers, stereoregularity in aldehyde polymerization can be achieved with simple initiators. Especially in the case of aldehydes with long side chains or bulky side groups such as the trichloromethyl group in chloral, stereoregular, isotactic polymers are readily formed. In the case of chloral relative weak nucleophilic anions such as a chloride ion of tetraalkyl ammonium or tetraalkyl phosphonium salts are good initiators to form isotactic polymer.

Perhaloacetaldehydes could be prepared with a wide range of size and shape of the side groups and their 
polymerization could be studied under a variety of relatively simple reaction conditions.

\section{Bromoaldehyde polymers}

Polybromal. Several attempts have been made in the past by us to polymerize bromal but without success. We have recently developed an improved method for the purification of bromal which could then be polymerized with pyridine as the initiator at $-78^{\circ}$.

Earlier work on the polymerization of bromal using lithium tertiary butoxide and triphenylphosphine was unsuccessful. Traditionally, bromal as received from Eastman Kodak Company was carefully distilled under reduced pressure in a spinning band column. As the distillation proceeded, a faint brown color developed even in the "purest" samples. Triphenylphosphine reacts with bromal to form the triphenyl dibromovinyloxy phosphonium bromide. As a consequence, the initiator for the bromal polymerization was believed to be bromide, an anion which was known not to be very effective in chloral polymerization.

We have now developed conditions for a more sensitive gas chromotographic analysis of bromal and have found that three impurities are present in commercial bromal samples one or more of these impurities was the cause of the inability of the aldehyde to polymerize even when the sample was treated with a substantial amount of initiator. In connection with a different aspect of our work on the preparation of haloaldehydes, we found that treatment of bromal with $\mathrm{SbF}_{3}$ gives bromal which after distillation is and remains colorless. Gas chromatographic analysis showed that two of the impurities have been completely removed by this treatment and that the third peak of the three impurities was reduced to less than $0.03 \%$.

When the sample of purified bromal was treated with about $2 \mathrm{~mol} \%$ of pyridine at $-78^{\circ}$ for $72 \mathrm{hr}$, a gel was obtained which was treated with methanol and gave a slightly yellow powder in $46 \%$ yield (Table 2).

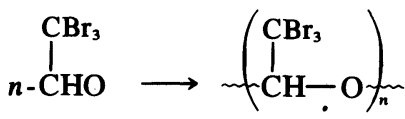

Table 2. Bulk polymerization of bromochloroacetaldehydes

\begin{tabular}{llrll}
\hline Perhaloaldehyde & $\begin{array}{c}\text { Initiator } \\
\text { type }\end{array}$ & $\begin{array}{c}\text { Polymerization } \\
\text { Time } \\
(\mathrm{hr})\end{array}$ & $\begin{array}{c}\text { Temperature } \\
\left({ }^{\circ} \mathrm{C}\right)\end{array}$ & $\begin{array}{c}\text { Polymer } \\
\text { yield } \\
(\%)\end{array}$ \\
\hline $\mathrm{BrCCl}_{2} \mathrm{CHO}$ & pyridine & 3 & -30 & 72 \\
& $\mathrm{LiOC}\left(\mathrm{CH}_{3}\right)_{3}$ & 3 & -30 & 80 \\
& $\mathrm{SbCl}_{5}$ & 170 & -30 & 58 \\
& $\mathrm{CF}_{3} \mathrm{SO}_{3} \mathrm{H}$ & 170 & -30 & 40 \\
$\mathrm{Br}_{2} \mathrm{CClCHO}$ & pyridine & 72 & -45 & 52 \\
& $\mathrm{SbCl}_{5}$ & 72 & -45 & 24 \\
$\mathrm{Br}_{3} \mathrm{CCHO}$ & pyridine & 72 & -78 & 46 \\
\hline
\end{tabular}

The polymer gave an i.r. spectrum which is very similar to the spectra of chloral and does not show any absorption in the carbonyl region (Fig. 11). Strong absorption in the regions of $800-1200 \mathrm{~cm}^{-1}$ indicate the presence of ether linkages. Elemental analysis also indicate that polybromal was obtained. Calcd for $\left(\mathrm{C}_{2} \mathrm{HOBr}_{3}\right)_{n}, \mathrm{Br}$, 85.38. Found: $\mathrm{Br}$, 85.11. Pyridine is the only initiator which gave reliably polybromal with our new polymerization grade bromal. Other anionic and cationic initiators have not been effective until now.

Bromal copolymerized with phenyl isocyanates with pyridine as the initiator at $-78^{\circ}$. The copolymer yields varied depending on actual copolymer composition but copolymers with up to $50 \mathrm{~mol} \%$ of phenylisocyanate have been isolated (Table 4).

Polydibromochloroacetaldehyde. Dibromochloroacetaldehyde was prepared in a sequence of steps starting with monochloroacetaldehyde diethyl acetal. The compound was brominated and treated with sulfuric acid whereby crude dibromochloroacetaldehyde separated as a heavy brownish oil. After additional treatment with sulfuric acid the compound was distilled from $\mathrm{P}_{2} \mathrm{O}_{5}$. Very careful redistillation gave polymer grade dibromochloroacetaldehyde which according to $\mathrm{GC}$ analysis contained about $0.1 \%$ of impurities.

When initiated with pyridine or $\mathrm{SbCl}_{5}$ at $-45^{\circ}$ a polymer was obtained which was worked up after $72 \mathrm{hr}$ and yielded $50 \%$ of a slightly yellow insoluble powder (Table 2).

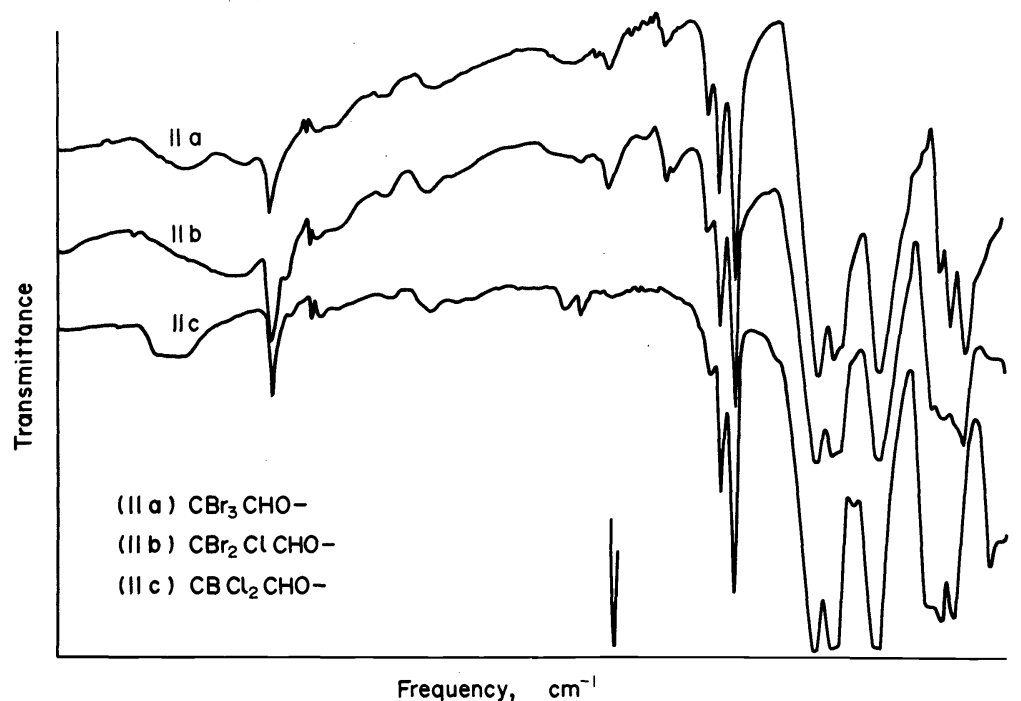

Fig. 11. I.R. spectra of polyhaloaldehydes: (KBr pellets). (a) Polybromal; (b) Polydibromochloroacetaldehyde; (c) Polybromodichloroacetaldehyde. 


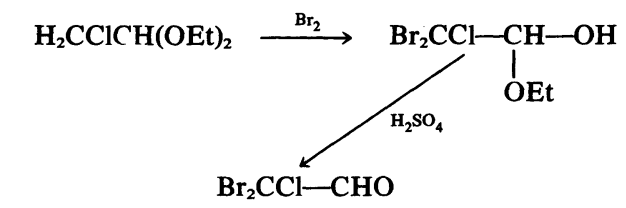

$\left.\underset{\mathrm{CHO}}{\mathrm{Br}_{2} \mathrm{CCl}} \stackrel{-45^{\circ} \text {, bulk }}{\stackrel{\text { pyridine initiator }}{\mathrm{Br}_{2} \mathrm{CCl}} !} \operatorname{fm}_{\mathrm{C} H}-\mathrm{O}\right)_{n}$

(9)

Elemental analysis: Calcd for $\left(\mathrm{C}_{2} \mathrm{HOBr}_{2} \mathrm{Cl}\right)_{n}, \mathrm{C}, 10.17$; $\mathrm{H}$, 0.42. Found: C, 10.38; H, 0.69.

The polymer gave an i.r. spectrum ( $\mathrm{KBr}$ pellet) which was very similar to the other bromochloroaldehyde polymers (Fig. 10). The spectrum was free from any substantial absorption in the carbonyl region and showed the typical C-O-C absorption.

Polybromodichloroacetaldehyde. Bromodichloroacetaldehyde could be prepared from dichloroacetaldehyde acetal. This reaction gives the aldehyde in low yield and insufficient purity. As a consequence, we used a sequence of steps which gave pure bromodichloroacetaldehyde starting from triphenylphosphine and its reaction product with chloral.

$$
\begin{aligned}
& \mathrm{Ph}_{3} \mathrm{P}-\mathrm{O}-\mathrm{CH}=\mathrm{CCl}_{2}+\mathrm{Br}_{2} \longrightarrow \\
& { }^{\oplus} \mathrm{Cl}{ }^{\ominus}
\end{aligned}
$$

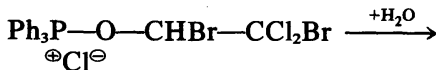

$$
\begin{aligned}
& \mathrm{Ph}_{3} \mathrm{PO}+\mathrm{BrCCl}_{2}-\mathrm{CHO}+\mathrm{HCl}+\mathrm{HBr} \\
& \sim 60 \%
\end{aligned}
$$

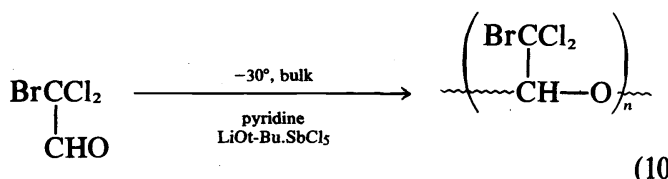

This compound was brominated and the dibromide purified and hydrolyzed to give bromodichloroacetaldehyde in good yield. After rectification from $\mathrm{P}_{2} \mathrm{O}_{5}$ the aldehyde was isolated and contained $0.1 \%$ of impurities according to gas chromatographic analysis.

The polymerization of bromodichloroacetaldehyde was achieved by treating the aldehyde with various anionic and cationic initiators at $-30^{\circ}$ for several hours. Pyridine, $\gamma$-collidine and lithium tertiary butoxide as well as trifluoromethyl sulfonic acid, $\mathrm{SbCl}_{5}$ and sulfuric acid gave polybromodichloroacetaldehvde in yields of up to $80 \%$ (Table 2). Elemental analysis: Calcd for $\left(\mathrm{C}_{2} \mathrm{HOBrCl}_{2}\right)_{n}$ : C, 12.52; H, 0.53. Found: C, 12.90; H, 0.69.

The infrared spectrum (KBr pellet) is in agreement with the proposed formula (Fig. 10).

Copolymers of bromodichloroacetaldehyde with chloral and with phenylisocyanates have also been prepared and characterized (Table 4).

Polydibromofluoroacetaldehyde. Dibromofluoroacetal- dehyde was prepared from bromal and $\mathrm{SbF}_{3}$. It was purified polymerized with pyridine and gave an insoluble polymer whose infrared spectrum is in agreement with the proposed formula

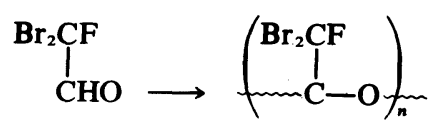

Elemental analysis: Calcd for $\left(\mathrm{C}_{2} \mathrm{HOBr}_{2}\right)_{n}, \mathrm{C}, 10.91 ; \mathrm{H}$, 0.45. Found: C, 11.06; H, 0.68 .

\section{E. Fluoroaldehyde polymers}

Polydifluorochloroacetalaldehyde. Polydifluorochloroacetaldehyde was prepared earlier and its polymerization described in a patent. ${ }^{23}$ We have worked out an effective way of preparing difluorochloralacetaldehyde by a sequence of steps starting with methyl difluorochloroacetate. Reduction with lithium aluminum hydride in ether at $-78^{\circ}$ gave the hydrate of difluorochloroacetaldehyde which was dehydrated with sulfuric acid and $\mathrm{P}_{2} \mathrm{O}_{5}$ and then polymerized.

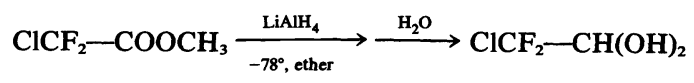

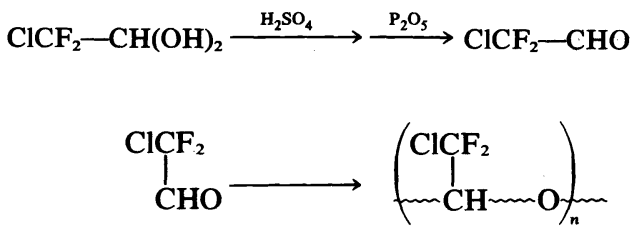

A number of anionic and cationic initiators proved to be effective for the polymerization of difluorochloroacetaldehyde, but lithium tertiary butoxide, triethylaluminum and triphenylphosphine were most effective (Table 3). ${ }^{25}$

Polymerization of difluorochloroacetaldehyde gave not only insoluble but also soluble polymer. Completely acetone soluble polymer was obtained with $\mathrm{SbCl}_{5}$ and with triphenylphosphine under certain conditions. In most other cases, polydiffuorochloroacetaldehyde is either

\begin{tabular}{|c|c|c|c|c|}
\hline Perhaloaldehyde & $\begin{array}{l}\text { Initiator } \\
\text { type }\end{array}$ & $\begin{array}{l}\text { Pol } \\
\text { Time } \\
\text { (hr) }\end{array}$ & $\begin{array}{l}\text { ymerization } \\
\text { Temperature } \\
\left({ }^{\circ} \mathrm{C}\right)\end{array}$ & $\begin{array}{l}\text { Polymer } \\
\text { yield } \\
\text { (\%) }\end{array}$ \\
\hline \multirow[t]{4}{*}{$\mathrm{F}_{2} \mathrm{CClCHO}$} & $\mathrm{Ph}_{3} \mathrm{P}$ & 1 & $\begin{array}{r}25 \\
-78\end{array}$ & $\begin{array}{l}55 \\
50\end{array}$ \\
\hline & $\mathrm{AlEt}_{3}$ & 1 & -78 & 91 \\
\hline & $\mathrm{LiOC}\left(\mathrm{CH}_{3}\right)_{3}$ & 1 & -78 & 92 \\
\hline & $\mathrm{SbCl}_{5}$ & 1 & -78 & 56 \\
\hline \multirow[t]{5}{*}{$\mathrm{FCCl}_{2} \mathrm{CHO}$} & $\mathrm{Ph}_{3} \mathrm{P}$ & 120 & -5 & 80 \\
\hline & $\mathrm{LiOC}\left(\mathrm{CH}_{3}\right)_{3}$ & 360 & 25 & 86 \\
\hline & pyridine & 1 & -78 & 12 \\
\hline & $\mathrm{SbCl}_{5}$ & 1 & -78 & 79 \\
\hline & $\mathrm{H}_{2} \mathrm{SO}_{4}$ & 46 & 25 & 82 \\
\hline
\end{tabular}
insoluble or a mixture of insoluble and soluble polymer.

Table 3. Polymerization of fluorochloroacetaldehyde 
Table 4. Copolymerization of perhaloaldehydes with phenylisocyanate

\begin{tabular}{lccccc}
\hline Perhaloaldehyde & $\begin{array}{c}\text { Phenylisocyanate } \\
(\mathrm{mol} \%)\end{array}$ & $\begin{array}{c}\text { Polymerization } \\
(\mathrm{hr})\end{array}$ & $\begin{array}{c}\text { Temperature } \\
\left({ }^{\circ} \mathrm{C}\right)\end{array}$ & $\begin{array}{c}\text { Initiator } \\
\text { type }\end{array}$ & $\begin{array}{c}\text { Copolymer } \\
\text { yield } \\
(\%)\end{array}$ \\
\hline $\mathrm{CCl}_{3} \mathrm{CHO}$ & $5-20$ & 1 & 0 & many & 85 \\
$\mathrm{CCl}_{2} \mathrm{BrCHO}$ & & & & & \\
$\mathrm{CBr}_{2} \mathrm{ClCHO}$ & 5 & 72 & -45 & pyridine & 72 \\
$\mathrm{CBr}_{3} \mathrm{CHO}$ & 5 & 72 & -78 & $\begin{array}{l}\text { pyridine } \\
\text { pyridine }\end{array}$ & 31 \\
& 10 & 72 & -78 & 50 \\
$\mathrm{CCl}_{2} \mathrm{FCHO}$ & 50 & 72 & -78 & pyridine & 74 \\
& 50 & 46 & -5 & $\mathrm{Ph}_{3} \mathrm{P}$ & 48 \\
\hline
\end{tabular}

Polyfluorodichloroacetaldehyde. Polyfluorodichloroacetaldehyde was prepared by a sequence of steps starting with methyl trichloroacetate, which was fluorinated with $\mathrm{SbF}_{3}$ to methyl fluorodichloroacetate. Lithium aluminum hydride reduction at $-78^{\circ}$ and hydrolysis of the product gave fluorodichloroacetaldehyde which was dehydrated with concentrated sulfuric acid and finally purified by distillation from $\mathrm{P}_{2} \mathrm{O}_{5}{ }^{26}$

$$
\mathrm{Cl}_{2} \mathrm{CF}-\mathrm{COOCH}_{3} \underset{-78^{\circ} \text {, ether }}{\stackrel{\mathrm{LiAlH}_{4}}{\longrightarrow}} \stackrel{\mathrm{H}_{2} \mathrm{O}}{\longrightarrow} \mathrm{Cl}_{2} \mathrm{CF}-\mathrm{CH}(\mathrm{OH})_{2}
$$

$\mathrm{Cl}_{2} \mathrm{CF}-\mathrm{CH}(\mathrm{OH})_{2} \stackrel{\mathrm{H}_{2} \mathrm{SO}_{4}}{\longrightarrow} \stackrel{\mathrm{P}_{2} \mathrm{O}_{5}}{\longrightarrow} \mathrm{Cl}_{2} \mathrm{CF}-\mathrm{CHO}$<smiles>CCOC(C)C(C)Cl</smiles>

Purified fluorodichloroacetaldehyde was polymerized with anionic and cationic initiators to give insoluble polyfluorodichloroacetaldehyde. A number of cationic initiators such as sulfuric acid, $\mathrm{TiCl}_{4}$ and $\mathrm{SbCl}_{5}$, gave good yields of polymer, but lithium tertiary butoxide and triphenylphosphine were the best initiators for this polymerization (Table 3 ).

Fluorodichloroacetaldehyde could be copolymerized with phenyl isocyanate and chloral.

\section{FUTURE PROBLEMS IN ALDEHYDE POLYMERIZATION}

Several problems in aldehyde polymerization are still awaiting further investigation. The general interest will depend on new developments and particularly on the introduction of additional commercial products. Much of the previous work on aldehyde polymerization was spurred by the commercialization of polyformaldehyde, the use of acetaldehyde for the development of stereospecific catalysts and finally the development of a monomer casting technique for chloral for the fabrication of novel nonflammable polymers.

(i) Future developments in aldehyde polymerization are foreseen in the success of polymerization of aldehydes with long side chains. This polymerization depends very much on the structure of individual monomers in solution and suggests a possible liquid crystal behavior of such solutions. If the molecular weight of these aldehyde polymers with long side chains could be improved, polymers with novel mechanical properties which might be useful as compatibilization agents in polymer mixtures are envisioned. (ii) Because of their much improved autoxidation resistance over polymers with short side chains, these polyaldehydes might give additional understanding of the diffusion of oxygen in microphase separated systems. (iii) Very little is known about copolymers of aliphatic polyaldehydes and it would be desirable to prepare copolymers, in order to improve molecular weight and other properties. (iv) Cryotachensic polymerization of chloral has led to the development of sequential polymerization of chloral and additional polymers which is the only way to prepare blends and crosslinked networks of polychloral. (v) Direct and complete capping by termination reactions described in this paper could lead to chloral polymers of commercially useful stability. (vi) The crystal structure of all the new haloaldehyde polymers should be investigated and the potential energy calculations should be carried out. When successfully completed, we should have a full understanding of the influence of the size and shape of the side groups on the perhaloacetaldehyde of the individual. (vii) Aliphatic aldehydes with functional groups will continue to be investigated in order to obtain materials with new properties.

\section{REFERENCES}

${ }^{1}$ O. Vogl, Polyaldehydes. Marcel Dekker, New York (1967).

${ }^{2} \mathrm{~J}$. Furukawa and T. Saegusa, Polymerization of Aldehydes and Oxides. J. Wiley (Interscience), New York (1963).

${ }^{3}$ J. C. Bevington, Quart. Rev. (Lond.) 6, 141 (1972).

${ }^{4}$ R. N. MacDonald, U.S. Patent 2,708,994 (1956).

${ }^{5}$ C. E. Schweizer, R. N. MacDonald and J. O. Punderson, J. Appl. Polym. Sci. 1, 158 (1959).

${ }^{6}$ O. Vogl, Makromol. Chem. 175, 1281 (1974).

${ }^{7}$ O. Vogl, in J. Macromol. Sci. C12(1), 109 (1975).

${ }^{8}$ O. Vogl, J. P. Kennedy and E. G. M. Tornqvist, Polymer Chemistry of Synthetic Elastomers. J. Wiley (Interscience), New York (1969).

${ }^{9}$ J. Furukawa, T. Saegusa and H. Fujii, Makromol. Chem. 44/46, 398 (1961); H. Tani and N. Oguni, J. Polym. Sci. B2, 123 (1964).

${ }^{10}$ O. Vogl, J. Polym. Sci. A1, 4621 (1964).

${ }^{11}$ H. Sumitomo and K. Kobayashi, J. Polym. Chem., Polym. Chem. 4, 907 (1966) and subsequent papers.

${ }^{12}$ O. Vogl, U.S. Patent 3,454,527 (1963).

${ }^{13}$ O. Vogl, H. C. Miller and W. H. Sharkey, Macromolecules 5, 658 (1972).

${ }^{14} \mathrm{H}$. Schechter and F. Conrad, J. Am. Chem. Soc. 72,3371 (1950).

${ }^{15}$ S. Temple and R. L. Thornton, J. Polym. Sci. A1(10), 709 (1972).

${ }^{16}$ I. Negulescu and O. Vogl, J. Polym. Sci., Polymer Letts 13, 17 (1975).

${ }^{17}$ I. Negulescu and O. Vogl, Abstr. of Papers, IUPAC Symp. Macromolecules, Madrid (1974). 
${ }^{18}$ T. Malkin and T. C. Tranter, J. Chem. Soc. 1178 (1951).

${ }^{19}$ O. Vogl and K. Hatada, J. Polym. Sci., PolymerLetts 13,603(1975). ${ }^{20}$ P. Kubisa and O. Vogl, Polymer J. (Japan) 7, 186 (1975).

${ }^{21}$ P. Kubisa and O. Vogl, Vysokomol. Soed. 17, 929 (1975).

${ }^{22}$ D. W. Lipp and O. Vogl, Abstr. of Papers, 6th NERM, No. 164, Burlington (1974).
${ }^{23}$ C. Woolf, U.S. Patent 2,870,213 (1959).

${ }^{24}$ B. Yamada and O. Vogl, Abstr. of Papers, 5th NERM, No. 121, Rochester (1973).

${ }^{25}$ B. Yamada and O. Vogl, Abstr. of Papers, Soc. Polym. Sci. (Japan), No. 178, Tokyo (1974). 